\title{
Systolic Blood Pressure is Positively Correlated With Headache Frequency: A 3-Month Follow-Up Study on 441 Migraine Patients
}

\section{Yen-Feng Wang}

Taipei Veterans General Hospital

\section{Yi-Shiang Tzeng}

Taipei Veterans General Hospital

Richard B. Lipton

Albert Einstein College of Medicine

Ai Seon Kuan

Taipei Veterans General Hospital

Shih-Pin Chen

Taipei Veterans General Hospital

Hao-min Cheng

Taipei Veterans General Hospital

Chen-Huan Chen

National Yang-Ming University

Shuu-Jiun Wang ( $\nabla$ sjwang@vghtpe.gov.tw)

Taipei Veterans General Hospital https://orcid.org/0000-0001-5179-5358

\section{Research article}

Keywords: Migraine, blood pressure, headache frequency, preventive treatment, prognosis

Posted Date: December 15th, 2020

DOI: https://doi.org/10.21203/rs.3.rs-126679/v1

License: (1) (1) This work is licensed under a Creative Commons Attribution 4.0 International License. Read Full License 
Systolic blood pressure is positively correlated with headache frequency:

A 3-month follow-up study on 441 migraine patients

Yen-Feng Wang ${ }^{1-3}$, Yi-Shiang Tzeng ${ }^{1}$, Richard B. Lipton ${ }^{4}$, Ai Seon Kuan ${ }^{2}$, 5-6, ShihPin Chen ${ }^{1-3,7}$, Hao-min Cheng ${ }^{2,6,8-10}$, Chen-Huan Chen ${ }^{2,6,8,10}$, and Shuu-Jiun Wang ${ }^{1-3}$

${ }^{1}$ Department of Neurology, Neurological Institute, Taipei Veterans General Hospital,

Taipei, Taiwan

${ }^{2}$ Faculty of Medicine, National Yang-Ming University School of Medicine, Taipei,

Taiwan

${ }^{3}$ Brain Research Center, National Yang-Ming University, Taipei, Taiwan

${ }^{4}$ Montefiore Headache Center, Albert Einstein College of Medicine, Bronx, New

York, United States

${ }^{5}$ Department of Neurosurgery, Neurological Institute, Taipei Veterans General

Hospital, Taipei, Taiwan

${ }^{6}$ Institute of Public Health, National Yang-Ming University, Taipei, Taiwan

${ }^{7}$ Division of Translational Research, Department of Medical Research, Taipei

Veterans General Hospital, Taipei, Taiwan

${ }^{8}$ Division of Cardiology, Department of Medicine, Taipei Veterans General Hospital, 
Taipei, Taiwan

${ }^{9}$ Center for Evidence-based Medicine, Taipei Veterans General Hospital, Taipei,

Taiwan

${ }^{10}$ Department of Medical Education, Taipei Veterans General Hospital, Taipei, Taiwan

Correspondence:

Shuu-Jiun Wang, MD, Department of Neurology, Neurological Institute, Taipei

Veterans General Hospital, No. 201, Sec. 2, Shi-Pai Road, Bei-Tou District, Taipei

City, Taiwan, 11217. Email: sjwang@vghtpe.gov.tw 


\section{ABSTRACT}

\section{Background}

Whether blood pressure (BP) per se correlates with headache frequency in migraine is uncertain.

\section{Methods}

Newly diagnosed migraine patients without anti-hypertensive or migraine preventive treatment were prospectively enrolled. Standardized BP measurement was done at each visit. Patients were dichotomized by the overall arithmetic mean of baseline systolic BPs (SBPs). Correlations between baseline SBPs and monthly headache days (MHDs), based on prospective headache diaries, were determined. Participants were treated as usual for 3 months. The associations between changes $(\Delta)$ in $\mathrm{BP}$ and in MHD at 3 months were examined.

\section{Results}

In total, $441(344 \mathrm{~F} / 97 \mathrm{M}$, mean age $39.1 \pm 10.3$ years) migraine patients were analyzed, including 197 (44.7\%) with above-average SBPs, i.e. $\geq 120.1 \mathrm{mmHg}$. There were significant correlations between SBPs and MHDs at baseline (Spearman's rho $=0.181$, $\mathrm{p}=0.012$ ) and between $\triangle \mathrm{SBPs}$ and $\triangle \mathrm{MHDs}$ at 3 months (Spearman's rho $=0.257$, $\mathrm{p}<0.001)$ in patients with above-average SBPs. In addition, $\mathrm{a} \geq 50 \% \mathrm{MHD}$ improvement increased the odds for 5-mmHg SBP reductions at 3 months (odds 
ratio $=1.70,95 \%$ confidence interval $=1.05-2.76, \mathrm{p}=0.032$ ).

\section{Conclusions}

SBP was associated with headache frequency in migraine patients and the association was stronger in those with higher SBP. The improvement in headache frequency

paralleled that in SBP, although the underlying mechanisms remain to be determined.

\section{Keywords}

Migraine, blood pressure, headache frequency, preventive treatment, prognosis 


\section{Introduction}

The association between blood pressure (BP) and migraine has been an issue of debate in the literature and also in the daily clinical practice, especially when it comes to the association between increased risks for cerebrovascular and cardiovascular events and migraine (1-5). The association between high BP and headache was first reported in the early twentieth century (6), although subsequent reports were inconsistent (7-15). Some studies reported that migraine was associated with an increased risk not only for co-morbid hypertension $(8,9)$, but also for developing hypertension during follow-up $(10,15)$. On the other hand, it was also reported that patients with migraine had lower systolic and pulse pressures, but higher diastolic pressure when compared with those without (7), and migraine was more common among normotensive individuals than hypertensive patients (16). However, most of these reports examined the prevalence or incidence of migraine within groups with or without hypertension. Whether BPs per se could have an impact on headache frequency in patients with migraine, or vice versa, has rarely been investigated.

Whether the outcomes of BP and headache in migraine are correlated with each other is uncertain. Based on cross-sectional studies, hypertension was believed to be a risk factor for migraine chronification (17-19), although longitudinal data have been scarce. However, some insights could be derived from migraine preventive or anti- 
hypertensive treatment. Some anti-hypertensive agents are also effective in migraine prophylaxis (20-24). Besides, a meta-analysis of clinical trials on anti-hypertensive agents reported a lower incidence of headache reported as an adverse event in the treatment group than in the placebo group (25). These findings suggest that the outcomes of headache and BP could be correlated, although further clarification is necessary.

The objectives of the present study were to investigate the association between headache frequency and BP per se in migraine patients, as well as the association between the outcomes of headache and BP at 3 months.

\section{Methods}

\section{$\underline{\text { Participants }}$}

This was a prospective study enrolling newly diagnosed migraine patients without prior treatment with migraine preventive or anti-hypertensive agents from the Headache Clinic of Taipei Veterans General Hospital. The diagnosis of migraine was made based on the criteria of the third edition of the International Classification of Headache Disorders (26). Patients were eligible at their first visits if they were (a) willing to participate in the study, (b) aged 20 or above, (c) diagnosed as migraine without aura or migraine with aura, and (d) occurrence of migraine before age 50. Patients were excluded if they had (a) an acute headache with a history of $<1$ month, 
(b) a secondary headache disorder, (c) a history of use of migraine preventive

medications or anti-hypertensive drugs within 3 months before enrollment, and (d)

physical or mental disorders precluding the completion of detailed history taking.

Eligible patients were enrolled in the study and were asked to keep a headache diary,

and a headache day was defined as a calendar day with a headache lasting $\geq 4$ hours.

They received preventive treatment at the discretion of the treating physicians.

Patients were included in the final analyses if they had at least one follow-up clinic

visit within 3 months. The study protocol was approved by the Institutional Review

Board at the Taipei Veterans General Hospital (IRB-TPEVGH No. 2018-10-008A).

All participants provided informed consent prior to participation.

\section{$\underline{\text { Blood pressure measurement }}$}

Blood pressures (BPs) were measured at each visit using a standard protocol. After having a rest in a quiet room for $>5$ minutes, two BP readings were obtained by a research assistant using a non-invasive stand-alone BP monitor (WatchBP Office Central, Microlife Corp., Taipei, Taiwan). The averaged values of the two BP readings, including systolic BPs (SBP), diastolic BPs (DBPs), and mean arterial pressures (MAPs), were used for analyses. The patients were categorized as having above- or below-average SBPs if their baseline SBPs were higher or lower, respectively, than the arithmetic mean value of the baseline SBPs of the entire study population. Identical 
procedures were used to measure BP at the follow-up visit at 3 months.

\section{$\underline{\text { Statistical analysis }}$}

Baseline characteristics between the above- and below-average SBP groups were compared by using student's t test for continuous variables or Mann-Whitney U test if the variables were not normally distributed, and by using chi-square test for categorical variables. The changes in $\mathrm{SBP}(\triangle \mathrm{SBP})$ and the change in the number of monthly headache day (MHD) ( $\triangle \mathrm{MHD}$ ) at 3 months were determined, and missing data were imputed by using the last-observation-carried-forward (LOCF) method.

Paired t test was used to compare the differences between baseline and at 3 months. As the null hypothesis of normality of our SBP and MHD data was rejected by the Shapiro-Wilk test, Spearman's rho statistics was used to examine the correlations between SBPs and the numbers of MHDs at baseline and between the changes in SBPs $(\triangle \mathrm{SBP})$ and the changes in MHDs $(\triangle \mathrm{MHDs})$ at 3 months. The patients in the above- and below-average SBP groups were further divided into higher and lower MHDs, as well as SBPs, by the medians within that group. Logistic regression modeling was carried out to compare the odds of having higher SBPs between patients with higher and lower MHDs at baseline, and to estimate the odds ratios (ORs), as well the 95\% confidence intervals (CIs), for having 5-mmHg improvement in SBP (27) at 3 months between patients with and without $\geq 50 \%$ reduction in MHD. 
Potential confounders were controlled, including age, sex, body mass index (BMI), monthly days of analgesic use at baseline (days/month), headache severity at the time of BP measurement (0-10/10 on numerical rating scale), analgesic use on the day of clinical visit, baseline BP status ( $<$ mean $/ \geq$ mean), and preventive treatment during the follow-up period. All statistical analyses were carried out by using IBM SPSS Statistics for Windows, version 24.0 (IBM Corp., Armonk, NY, USA. Statistical significance was defined as a two-sided $\mathrm{p}$ of $<0.05$.

\section{Results}

Baseline characteristics (table 1)

In total, 600 migraine patients $(470 \mathrm{~F} / 130 \mathrm{M}$, mean age $40.0 \pm 10.7$ years, mean headache frequency $9.8 \pm 7.7$ days per month, mean SBP $119.9 \pm 18.6 \mathrm{mmHg}$ ) were enrolled. Of them, $159(122 \mathrm{~F} / 37 \mathrm{M}$, mean age $37.8 \pm 10.8)$ were excluded for the use of anti-hypertensive agents $(n=5)$ or preventive medications $(n=51)$, or loss to follow-up after the initial visit $(\mathrm{n}=103)$, and 441 (344F/97M, mean age $39.1 \pm 10.3$ years) (73.5\%) were included in the final analyses. At baseline, the headache frequency was 9.8 \pm 7.7 MHDs, and the SBP, DBP, and MAP were 120.1 $\pm 18.9,75.8 \pm 11.6$, and $90.6 \pm 13.4 \mathrm{mmHg}$, respectively. The patients were then divided into two groups by the arithmetic mean of baseline SBPs of the entire population, i.e. $\geq 120.1 \mathrm{mmHg}(\mathrm{n}=192$, 
$43.5 \%)$ and $<120.1 \mathrm{mmHg}(\mathrm{n}=249,56.5 \%)$. Patients in the above-average SBP group were older $(42.2 \pm 10.2$ vs. $36.7 \pm 9.8$ years, $\mathrm{p}<0.001)$ and less likely to be a woman (62.0\% vs. $90.4 \%, \mathrm{p}<0.001)$ (table 1$)$. Besides, they had higher BMIs $(25.0 \pm 4.1$ vs. 21.3 $\pm 2.7, \mathrm{p}<0.001)$ and more monthly days of analgesic use $(7.4 \pm 7.8$ vs. $5.5 \pm 6.4$ days, $\mathrm{p}=0.010)$; whereas the headache frequencies (9.9 \pm 8.3 vs. 9.7 \pm 7.3 MHDs, $\mathrm{p}=0.598)$ and the proportions of migraine with aura $(9.9 \%$ vs. $10.0 \%, \mathrm{p}=0.960)$, nonsteroidal anti-inflammatory drug (NSAID) use (48.4\% vs. $43.0 \%, \mathrm{p}=0.253)$, and triptan use $(6.8 \%$ vs. $7.2 \%, \mathrm{p}=0.852)$ were comparable.

Correlations between blood pressures and headache frequency at baseline

Overall, headache frequency increased with baseline SBP (figure 1) in the entire study population. There was a positively correlation between MHD and SBP in patients with above-average baseline SBPs (Spearman's rho=0.181, $\mathrm{p}=0.012$ ), but not in those with below-average baseline SBPs (Spearman's rho=0.029, $\mathrm{p}=0.646$ ) (figure 1A, table 2). On the other hand, there was no correlation between SBP and headache severity at the time of BP measurement at baseline (Spearman's rho=0.051, $\mathrm{p}=0.290$ ). The correlations were similar but weaker in DBPs and MAPs (table 2). Patients with higher MHDs were more likely to have higher SBPs after controlling for potential confounders, although the association was present only in patients with above-average SBPs $(\mathrm{OR}=2.31,95 \% \mathrm{CI}=1.17-4.56, \mathrm{p}=0.017)$, but not in those with below-average 
SBPs $(\mathrm{OR}=1.21,95 \% \mathrm{CI}=0.66-2.21, \mathrm{p}=0.534)($ Figure 1B).

Treatment and clinical outcomes at 3 months

The average interval from baseline to 3 -month follow-up was $2.1 \pm 0.9$ months.

During the 3-month follow-up period, 223 patients (50.6\%) were treated with preventives, including 135 (60.5\%) with flunarizine, 87 (39.0\%) with beta-blockers, $20(9.0 \%)$ with antiepileptics, $17(7.6 \%)$ with candesartan or lisinopril, $9(4.0 \%)$ with onabotulinumtoxinA, and $6(2.7 \%)$ with tricyclics or serotonin-noradrenalin reuptake inhibitors. Among these patients, $47(21.1 \%)$ used more than one category of preventive agents. Besides, 415 (94.1\%) patients received acute medications, including $356(85.8 \%)$ with triptans, 129 (31.1\%) with NSAIDs, 59 (14.2\%) with ergots, $2(0.5 \%)$ with acetaminophen, and $2(0.5 \%)$ with acetaminophen/tramadol. Overall, the headache frequencies decreased at 3 months (9.8 \pm 7.7 vs. $7.9 \pm 6.6$ MHDs, $\mathrm{p}<0.001)$, as did SBPs $(120.1 \pm 18.9$ vs. $114.6 \pm 15.5 \mathrm{mmHg}, \mathrm{p}<0.001)$

Association between changes in systolic blood pressures and headache frequencies at 3 months

Overall, the extent of MHD improvement was greater in patients with higher baseline SBPs (figure 2A). There was a statistically significant correlation between $\Delta \mathrm{MHDs}$ and $\triangle \mathrm{SBP}$ (Spearman's rho $=0.114, \mathrm{p}=0.016$ ), which was derived from patients with above-average SBPs at baseline (Spearman's rho $=0.257, \mathrm{p}<0.001$ ), but 
not from those with below-average SBPs (Spearman's rho $=0.052, \mathrm{p}=0.410$ ) (figures 2B \& 2C).

In the entire study population, patients with a $\geq 50 \%$ decrease in MHDs were more likely to have SBP reductions by $5 \mathrm{mmHg}(\mathrm{OR}=1.63$ [1.05-2.55], $\mathrm{p}=0.031)$ when compared with those without, after controlling for potential confounders (table 3). Besides, the findings were consistent even when baseline SBP status $(<$ mean $/ \geq$ mean $)$ (model 2$)$ or headache severity at the time of BP measurement (model 3) were further controlled (table 3). The findings were consistent for 3-mmHg and $7-\mathrm{mmHg}$ reductions, and were more pronounced in those treated with preventive medications in exploratory analysis (supplementary table).

\section{Discussion}

In this large prospective clinic-based cohort $(n=441)$, the relationship between SBPs and headache frequencies was demonstrated in newly diagnosed preventiveand anti-hypertensive-naïve migraine patients. At baseline, SBPs were correlated with headache frequencies (Spearman's rho=0.181, $\mathrm{p}=0.012$ ), and patients with higher MHDs were more likely to have higher SBPs $(\mathrm{OR}=2.31,95 \% \mathrm{CI}=1.17-4.56$, $\mathrm{p}=0.017$ ). At 3-month follow-up, the decrease in headache frequency paralleled that in SBP. The findings were more pronounced in patients with above-average SBPs, i.e. 
$\geq 120.1 \mathrm{mmHg}$. More importantly, the findings remained after controlling for potential confounders, including headache severity at the time of BP measurement and presence or absence of analgesic use.

One of the strengths of the present study is the relatively large sample size. In the present study, SBP per se and headache frequency, rather than the presence or absence of hypertension or migraine, were collected and analyzed. We assessed the association between a physiological parameter and attack frequency, not merely the association between two diagnostic categories. Such in-depth analysis would not be feasible without such data abundance. Secondly, the data are of high quality. Migraine diagnoses were made by in-person clinical evaluations by headache specialists. BP was collected prospectively using a standard protocol. The number of MHDs were assessed with prospective daily diaries. In addition, the prospective and longitudinal nature of the present study reduced recall bias, which could be common for retrospective or cross-sectional designs.

In the baseline analysis of the present study, headache frequencies were correlated with SBPs in migraine patients, consistent with some prior reports, which provided evidence in favor of a positive association between migraine and hypertension (8-10, 13-15, 27-30). Some prior studies have reported an inverse association $(7,11,12,31)$ or even the absence of association (32). The discrepancies 
could be, at least in part, attributed to the differences in study populations and methodologies. Most of the published studies were population-based studies, and investigated the prevalence or incidence of migraine and hypertension diagnoses rather than the disease severities. In contrast, the current study examined the association between headache frequency and SBP per se in migraine patients using both cross-sectional and longitudinal data, and patients with above- and belowaverage baseline SBPs were analyzed separately. Otherwise it would not be easy to pick up the correlation between SBP and headache frequency as the overall association in the entire study sample was not significant. On the other hand, even though theoretically acute medications could have a role, the proportions of patients using NSAIDs or triptans were comparable between patients with above- and belowaverage baseline SBPs (table 1). Besides, the association remained after controlling for potential confounders (supplementary figure). However, the associations were not strong. It could not be excluded that part of the associations was derived from patients with SBPs much higher than the rest of the study population. Nevertheless, patients with very high SBPs, i.e. $\geq 160 \mathrm{mmHg}$ constituted only $3.4 \%(\mathrm{n}=15)$ of the study population, and might not have a substantial contribution to the finding. Therefore, it is likely that the association was present in patients with above-average SBPs, and could be more pronounced in those with very high SBPs. Further studies are needed 
to provide evidence for or against such an association.

During follow-up, there was a trend toward greater improvement in headache frequency in those with higher baseline SBPs (figure 2A). There was a positive correlation between $\triangle \mathrm{SBP}$ and $\triangle \mathrm{MHD}$, especially in patients with above-average SBPs at baseline (figures 2B \& 2C). Our findings seemed incongruent with those of the HUNT studies, which concluded with an inverse relationship between BPs and migraine $(12,31)$. In the HUNT studies, elevated SBPs and pulse pressures were associated with decreased odds of having migraine at 11-years' follow-up. Differences in study design (clinic-based vs. population-based), outcome measure (change in headache frequency in migraine patients vs. change in migraine prevalence), and follow-up duration (3 months vs. 11 years) could account for these differences. More importantly, a substantial proportion $(50.6 \%)$ of patients in the present study were treated with preventive medications. Therefore, the finding of the present study could not be compared with those of the HUNT studies directly. However, our findings could have a more practical impact for headache clinicians as the results were derived from a clinical setting.

There was accumulating evidence for increased risks of cardiovascular and cerebrovascular events in migraine patients when compared to healthy controls (1-5), and elevated BP might be a potential missing link between migraine and vascular 
events. In the present study, $a \geq 50 \%$ improvement in headache frequency was associated with an increased likelihood of SBP reduction at 3 months in the present study. On the other hand, even though migraine with aura was commonly mentioned as a risk factor for vascular events (4), the proportions of patients with aura were comparable between above- and below-average baseline SBP groups, which could suggest that BP might not have a role in the association between migraine aura and vascular risks. Even though SBP was selected as the primary BP parameter in the present study, the relative significance of different BP parameters as to the underlying pathophysiology remains unknown. Recently, it was reported that there was a positive correlation between central SBP and the volume of cerebral white matter hyperintensities (28), which could suggest that SBP could play a more important role. On the other hand, some studies suggested the potential roles of endothelial dysfunction (35-37) and systemic inflammation (38-41). Endothelial dysfunction has long been believed to participate in the pathophysiology of hypertension and vascular diseases (35), and its role in migraine is drawing increasing attention in recent years (36). In fact, according to a pilot study from our group, migraine patients had increased circulating endothelial-specific microRNAs (37), two of which, i.e. miR155 and miR-126, were related to a number of chronic pain disorders (42). Hypertension could lead to chronic systemic inflammation related to vessel wall shear 
stress, oxidative stress, and endothelial dysfunction, which could result in atherosclerosis and target-organ damage $(38,39)$. There is also some evidence suggesting the involvement of inflammatory cytokines in the pathogenesis of migraine $(40,41)$. However, whether endothelial dysfunction or inflammation participates in the association between BPs and migraine frequency warrants further clarification.

Our study had some limitations. First, the current study was a clinic-based study, and the generalizability of the findings may be limited due to concerns about selection bias. However, a relatively large cohort was analyzed and the data were collected in a standardized fashion, both of which could potentially minimize selection bias. Besides, a well-structured referral system is still being developed in the healthcare system in Taiwan, and a substantial proportion of patients in our headache clinic came in directly without referral. Therefore, even though this was a clinic-based study, the data collected could reflect the situation in the field to a certain extent. Secondly, as a real-world observation study, there was not a predetermined treatment protocol, and the treatment was determined at the discretion of the treating physicians. Whether different treatment strategies could have different impacts on our findings is unknown. However, the findings remained after controlling for these potential confounders. Third, SBP was used as the primary BP parameter based on the 
estimated effect size and statistical significance, and whether SBP, as compared with other BP parameters, might have a more important role remains to be further studied. Fourth, the classification of status of baseline SBPs was based on the measured SBPs at the first visit. It could be possible that some of patients belonging to the belowaverage SBP group could actually had above-average SBP before or after BP measurement at the first visit, or vice versa. However, the effect of misclassification bias could be reduced by the relatively large sample size $(n=441)$ of the present study. More importantly, such misclassification would result in underestimation rather than overestimation of the association. Fifth, the 3-month follow-up period may not be adequate to have a complete assessment of headache outcome, given the fluctuating nature of migraine as well as BPs (43). Finally, BP measurement was carried out on the left side, rather than on both sides, and it could not be excluded that a minority of patients could have conditions that could have an impact on the measured BP, such as subclavian steal syndrome.

\section{Conclusions}

The present study identified an association between SBPs and headache frequencies in migraine patients, particularly in those with above-average SBPs at baseline. This could indicate that SBPs might be an indicator of disease severity of migraine, especially when taking into account that the SBP measured was correlated with the 
headache frequency in the previous month. Besides, the change in SBP was in parallel with that in headache frequency. However, the underlying mechanisms remain to be determined. Further studies are needed to verify these findings.

\section{Abbreviations}

BP: blood pressure

BMI: body-mass index

CI: confidence interval

DBP: diastolic blood pressure

LOCF: last-observation-carried-forward

MAP: mean arterial pressure

MHD: monthly headache days

NSAID: non-steroidal anti-inflammatory drug

OR: odds ratio

SBP: systolic blood pressure

\section{Declarations}

\section{Ethics approval and consent to participate}

The study protocol was approved by the Institutional Review Board at the Taipei 
Veterans General Hospital (IRB-TPEVGH No. 2018-10-008A). All participants

provided informed consent prior to participation.

\section{Consent for publication}

Not applicable

\section{Availability of data and materials}

The datasets used and/or analyzed during the current study are available from the corresponding author on reasonable request.

\section{Competing interests}

YFW has received honoraria as a speaker from Taiwan branches of AbbVie, Eli Lilly,

Novartis, Pfizer, Sanofi, and UCB. He has received research grants from the Taiwan

Ministry of Science and Technology, and Taipei Veterans General Hospital. SJW has

served on the advisory boards of Eli Lilly, Daiichi-Sankyo, and Taiwan Novartis. He has

received honoraria as a moderator from AbbVie, Bayer, Eisai, Eli Lilly, and Pfizer. He has

received research grants from the Taiwan Ministry of Science and Technology, National

Yang-Ming University, Taipei Veterans General Hospital, Taiwan Headache Society,

Taiwan Pfizer, Taiwan Novartis, and Taiwan Eli Lilly. YHT, RBL, ASK, SPC, HMC, and 
CHC declared no relevant conflict of interest.

\section{Funding}

The authors disclosed receipt of the following financial support for the research, authorship, and/or publication of this article: Taiwan Ministry of Science and Technology [109-2314-B-075 -054] (to YFW), [MOST 107-2314-B-010-021, 1082314-B-010-022-MY3] (to SPC), and [MOST 108-2321-B-010-014 -MY2, 108-2321B-010 001, and 108-2314-B-010-023-MY3] (to SJW); Taiwan Ministry of Health and Welfare [MOHW104-TDU-B-211-113-003, MOHW106-TDU-B-211-113001] (to HMC); National Yang-Ming University (E107F-M01-0501) (to HMC); and Taipei Veterans General Hospital [VGH 108-C-092 and VGH 109-C-096] (to YFW), [VGH109-D52-001-MY3-1] (to SPC), and [VGH-109-D52-002-MY3-1] (to CHC); this work was supported by the Brain Research Center, National Yang-Ming University from The Featured Areas Research Center Program within the framework of the Higher Education Sprout Project by the Ministry of Education (MOE) in Taiwan, and Yin Yen-Liang Foundation Development and Construction Plan of National YangMing University School of Medicine. The funders had no role in study design, data col-lection and analysis, decision to publish, or preparation of the manuscript. 


\section{Authors' contributions}

YFW and SJW conceived and designed the study, acquired the data, and completed

the first draft. YFW, YST, ASK, and SJW analyzed the data. RBL, SPC, HMC, and CHC participated in the discussion and critical revision of the manuscript. The authors have read and approved the manuscript.

\section{Acknowledgements}

The authors would like to thank the study participants for their contributions.

\section{Reference}

1. Kurth T, Winter AC, Eliassen AH, Dushkes R, Mukamal KJ, Rimm EB, et al.

Migraine and risk of cardiovascular disease in women: prospective cohort study. BMJ. 2016;353:i2610.

2. Kurth T, Gaziano JM, Cook NR, Bubes V, Logroscino G, Diener HC, et al. Migraine and risk of cardiovascular disease in men. Arch Intern Med. 2007;167(8):795-801.

3. Adelborg K, Szepligeti SK, Holland-Bill L, Ehrenstein V, Horvath-Puho E, Henderson VW, et al. Migraine and risk of cardiovascular diseases: Danish population based matched cohort study. BMJ. 2018;360:k96.

4. Schurks M, Rist PM, Bigal ME, Buring JE, Lipton RB, Kurth T. Migraine and cardiovascular disease: systematic review and meta-analysis. BMJ. 2009;339:b3914.

5. Spector JT, Kahn SR, Jones MR, Jayakumar M, Dalal D, Nazarian S. Migraine headache and ischemic stroke risk: an updated meta-analysis. Am J Med.

2010;123(7):612-24.

6. Janeway TC. A clinical study of hypertensive cardiovascular disease. Arch Intern Med. 1913;12:755-98.

7. Gudmundsson LS, Thorgeirsson G, Sigfusson N, Sigvaldason H, Johannsson M. 
Migraine patients have lower systolic but higher diastolic blood pressure compared with controls in a population-based study of 21,537 subjects. The Reykjavik Study. Cephalalgia. 2006;26(4):436-44.

8. Bigal ME, Kurth T, Santanello N, Buse D, Golden W, Robbins M, et al. Migraine and cardiovascular disease: a population-based study. Neurology. 2010;74(8):628-35. 9. Scher Al, Terwindt GM, Picavet HS, Verschuren WM, Ferrari MD, Launer LJ. Cardiovascular risk factors and migraine: the GEM population-based study. Neurology. 2005;64(4):614-20.

10. Entonen AH, Suominen SB, Korkeila K, Mantyselka PT, Sillanmaki LH, Ojanlatva A, et al. Migraine predicts hypertension--a cohort study of the Finnish working-age population. Eur J Public Health. 2014;24(2):244-8.

11. Hagen K, Stovner LJ, Vatten L, Holmen J, Zwart JA, Bovim G. Blood pressure and risk of headache: a prospective study of 22685 adults in Norway. J Neurol Neurosurg Psychiatry. 2002;72(4):463-6.

12. Fagernaes CF, Heuch I, Zwart JA, Winsvold BS, Linde M, Hagen K. Blood pressure as a risk factor for headache and migraine: a prospective population-based study. Eur J Neurol. 2015;22(1):156-62, e10-1.

13. Gardener H, Monteith T, Rundek T, Wright CB, Elkind MS, Sacco RL. Hypertension and Migraine in the Northern Manhattan Study. Ethn Dis. 2016;26(3):323-30.

14. Cirillo M, Stellato D, Lombardi C, De Santo NG, Covelli V. Headache and cardiovascular risk factors: positive association with hypertension. Headache. 1999;39(6):409-16.

15. Rist PM, Winter AC, Buring JE, Sesso HD, Kurth T. Migraine and the risk of incident hypertension among women. Cephalalgia. 2018;38(12):1817-24.

16. Wiehe M, Fuchs SC, Moreira LB, Moraes RS, Fuchs FD. Migraine is more frequent in individuals with optimal and normal blood pressure: a population-based study. J Hypertens. 2002;20(7):1303-6.

17. Bigal ME, Sheftell FD, Rapoport AM, Tepper SJ, Lipton RB. Chronic daily headache: identification of factors associated with induction and transformation. Headache. 2002;42(7):575-81.

18. Buse DC, Manack A, Serrano D, Turkel C, Lipton RB. Sociodemographic and comorbidity profiles of chronic migraine and episodic migraine sufferers. J Neurol Neurosurg Psychiatry. 2010;81(4):428-32.

19. Gipponi S, Venturelli E, Rao R, Liberini P, Padovani A. Hypertension is a factor associated with chronic daily headache. Neurol Sci. 2010;31 Suppl 1:S171-3.

20. Schrader H, Stovner LJ, Helde G, Sand T, Bovim G. Prophylactic treatment of migraine with angiotensin converting enzyme inhibitor (lisinopril): randomised, 
placebo controlled, crossover study. BMJ. 2001;322(7277):19-22.

21. Tronvik E, Stovner LJ, Helde G, Sand T, Bovim G. Prophylactic treatment of migraine with an angiotensin II receptor blocker: a randomized controlled trial. JAMA. 2003;289(1):65-9.

22. Schuh-Hofer S, Flach U, Meisel A, Israel H, Reuter U, Arnold G. Efficacy of lisinopril in migraine prophylaxis--an open label study. Eur J Neurol. 2007;14(6):7013.

23. Diener HC, Gendolla A, Feuersenger A, Evers S, Straube A, Schumacher H, et al. Telmisartan in migraine prophylaxis: a randomized, placebo-controlled trial. Cephalalgia. 2009;29(9):921-7.

24. Stovner LJ, Linde M, Gravdahl GB, Tronvik E, Aamodt AH, Sand T, et al. A comparative study of candesartan versus propranolol for migraine prophylaxis: $A$ randomised, triple-blind, placebo-controlled, double cross-over study. Cephalalgia. 2014;34(7):523-32.

25. Law M, Morris JK, Jordan R, Wald N. Headaches and the treatment of blood pressure: results from a meta-analysis of 94 randomized placebo-controlled trials with 24,000 participants. Circulation. 2005;112(15):2301-6.

26. Society HCCotlH. Headache Classification Committee of the International Headache Society (IHS) The International Classification of Headache Disorders, 3rd edition. Cephalalgia. 2018;38(1):1-211.

27. Bundy JD, Li C, Stuchlik P, Bu X, Kelly TN, Mills KT, et al. Systolic Blood Pressure Reduction and Risk of Cardiovascular Disease and Mortality: A Systematic Review and Network Meta-analysis. JAMA Cardiol. 2017;2(7):775-81.

28. Cheng $\mathrm{CY}$, Cheng HM, Chen SP, Chung $\mathrm{CP}$, Lin $\mathrm{YY}, \mathrm{Hu} \mathrm{HH}$, et al. White matter hyperintensities in migraine: Clinical significance and central pulsatile hemodynamic correlates. Cephalalgia. 2018;38(7):1225-36. 
Figures
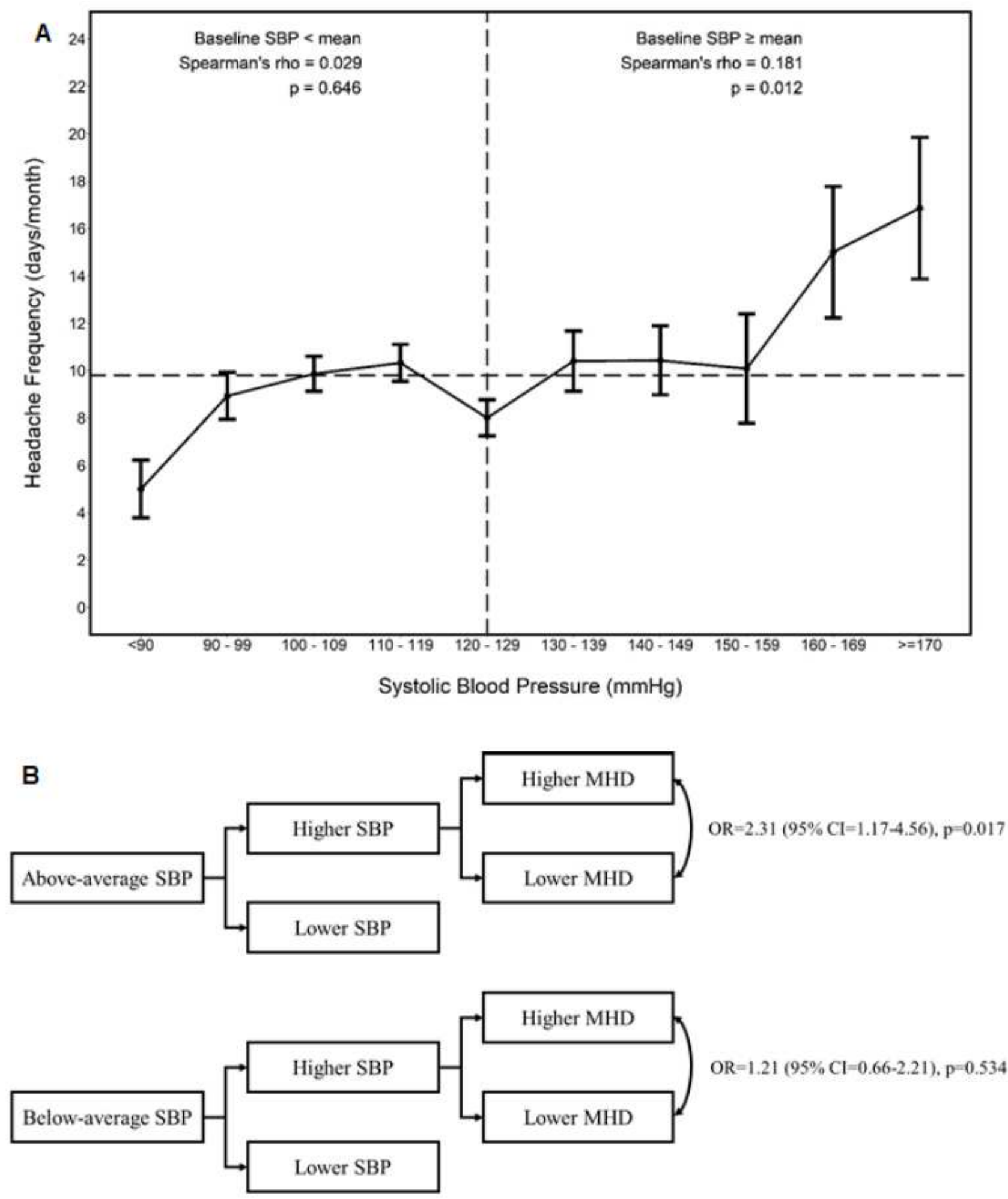

\section{Figure 1}

Association of headache frequency and systolic blood pressure at baseline A. Mean headache frequency (number of monthly headache days, MHD) \pm standard error stratified by systolic blood pressure (SBP). Dashed line indicates the overall mean headache frequency in the entire study population, i.e. 9.7 days 
per month, and overall mean systolic blood pressure, i.e. $120.1 \mathrm{mmHg}$ B. The associations between monthly headache frequencies and baseline blood pressures, stratified by baseline SBPs. Adjusted for age, gender, body-mass index, monthly days of analgesic use at baseline (days/month), headache score on the day of clinic visit at baseline (0-10/10), and baseline BP status (< mean/ $\geq$ mean) Abbreviations: $\mathrm{Cl}=$ confidence interval, $\mathrm{DBP}=$ diastolic blood pressure, $\mathrm{MAP}=$ mean arterial pressure, $\mathrm{SBP}=$ systolic blood pressure
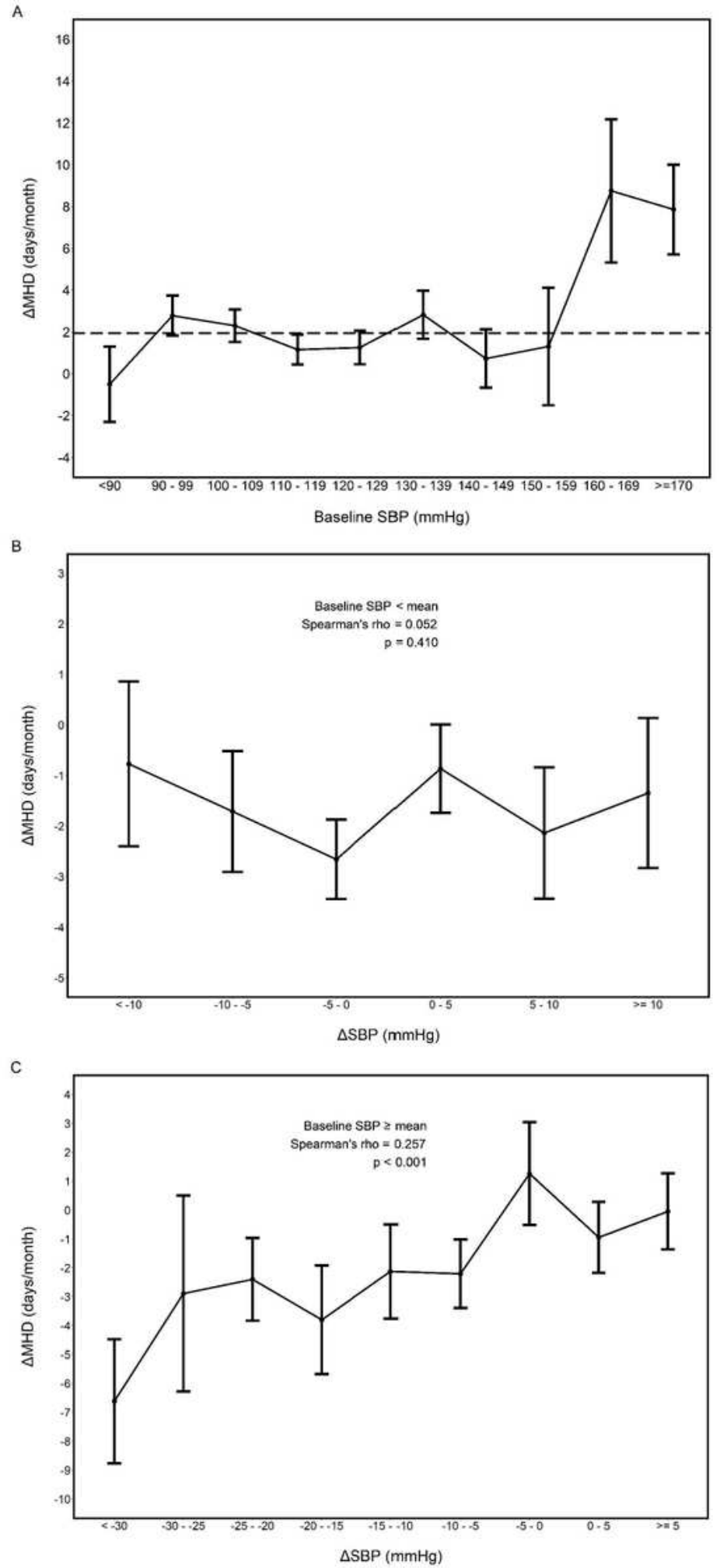

Figure 2 
Association of changes of headache frequency and systolic blood pressure at 3 months A. Patients with higher baseline SBP had greater improvement in the number of monthly headache days at 3 months $B$. There was no association between the change in SBP $(\triangle \mathrm{SBP})$ and that in the number of monthly headache days ( $\triangle \mathrm{MHDs}$ ) at 3 months in patients with below-average SBPs, i.e. $<120.1 \mathrm{mmHg}$ C. $\triangle \mathrm{SBP}$ was positively correlated with $\triangle M H D s$ in patients with above-average baseline SBPs, i.e. $\geq 120.1 \mathrm{mmHg}$

\section{Supplementary Files}

This is a list of supplementary files associated with this preprint. Click to download.

- tables201127.pdf 